\title{
Editorial: Kompetenzmodelle zur Erfassung individueller Lernergebnisse und zur Bilanzierung von Bildungsprozessen
}

\author{
Aktuelle Diskurse im DFG-Schwerpunktprogramm
}

Detlev Leutner $\cdot$ Eckhard Klieme $\cdot$ Jens Fleischer $\cdot$ Harm Kuper

\begin{abstract}
Zusammenfassung: Das Sonderheft umfasst elf Beiträge aus dem DFG-Schwerpunktprogramm „Kompetenzmodelle zur Erfassung individueller Lernergebnisse und zur Bilanzierung von Bildungsprozessen“. Ziel des Sonderhefts ist es, den Diskussionsstand des Schwerpunktprogramms hinsichtlich grundlegender Konzepte und Fragestellungen im Zusammenhang mit der Modellierung von Kompetenzen aufzuzeigen. Im ersten Beitrag werden die Struktur und die Forschungsbereiche des Schwerpunktprogramms vorgestellt sowie der aktuelle Forschungsstand skizziert. In den Beiträgen zwei bis elf werden in Form von Kurzbeiträgen Fragen und Problemstellungen diskutiert, die sich im Laufe der inhaltlichen Arbeit des Schwerpunktprogramms der letzten Jahre als besonders relevant erwiesen haben. Diese Beiträge stellen das Arbeitsergebnis eines Workshops der am Schwerpunktprogramm beteiligten Wissenschaftler/innen im Sinne von Positionspapieren dar.
\end{abstract}

Schlüsselwörter: Kompetenzmodelle $\cdot$ Kompetenzdiagnostik $\cdot$ DFG-Schwerpunktprogramm

(C) Springer Fachmedien Wiesbaden 2013

Prof. Dr. D. Leutner $(\bowtie) \cdot$ Dipl.-Psych. J. Fleischer

Institut für Psychologie, Universität Duisburg-Essen, 45117 Essen, Deutschland

E-Mail: detlev.leutner@uni-due.de

Dipl.-Psych. J. Fleischer

E-Mail: jens.fleischer@uni-due.de

Prof. Dr. E. Klieme

Deutsches Institut für Internationale Pädagogische Forschung, Bildungsqualität und Evaluation

Schloßstr. 29, 60486 Frankfurt am Main, Deutschland

E-Mail: klieme@dipf.de

Prof. Dr. H. Kuper

Erziehungswissenschaft und Psychologie, Freie Universität Berlin, Arnimallee 12, 14195 Berlin,

Deutschland

E-Mail: harm.kuper@fu-berlin.de 


\title{
Editorial: competence models for assessing individual learning outcomes and evaluating educational processes - current discourses within the DFG priority program
}

\begin{abstract}
This special issue comprises eleven articles from the DFG priority program "Competence Models for Assessing Individual Learning Outcomes and Evaluating Educational Processes". The special issue aims at showing the priority program's present state of discussion concerning essential concepts and questions related to the modeling of competencies. The first article describes the structure and research areas of the program and gives an overview on the current state of research. The short articles two to eleven discuss questions and problems which proved to be of particular importance during the work of the priority program in the last years. These articles constitute the result of a workshop of the participating scientists of the priority program in terms of position papers.
\end{abstract}

Keywords: Competence assessment $\cdot$ Competence models $\cdot$ DFG priority program

Die Modellierung und Messung von Kompetenzen spielt eine entscheidende Rolle bei der Optimierung von Bildungsprozessen sowie der Qualitätssicherung und Qualitätsentwicklung des Bildungswesens. Das DFG-Schwerpunktprogramm „Kompetenzmodelle zur Erfassung individueller Lernergebnisse und zur Bilanzierung von Bildungsprozessen“ (Klieme und Leutner 2006) hat sich zum Ziel gesetzt, die wissenschaftlichen Anstrengungen in diesem Bereich zu bündeln und zu koordinieren. Das DFG-Schwerpunktprogramm, welches 2007 eingerichtet wurde und 2012 in seine dritte und letzte zweijährige Förderphase gegangen ist, besteht aus 30 Projekten, die sich über Fachdisziplinen hinweg mit verschiedenen Aspekten der Modellierung und Messung von Kompetenzen befassen (vgl. Fleischer et al. 2012; Klieme et al. 2010; Koeppen et al. 2008). Für den Erfolg eines interdisziplinären Forschungsverbunds wie dem Schwerpunktprogramm - mit Beteiligung der Erziehungswissenschaft, der Psychologie und der Fachdidaktiken - ist eine Verständigung über die Bedeutung gemeinsam verwendeter Begrifflichkeiten von entscheidender Bedeutung. Das vorliegende Sonderheft umfasst elf Beiträge aus dem Kontext des DFG-Schwerpunktprogramms mit dem Ziel, den Diskussionsstand des Schwerpunktprogramms hinsichtlich grundlegender Konzepte und Fragestellungen im Zusammenhang mit der Modellierung von Kompetenzen vor dem Hintergrund des aktuellen Forschungsstands aufzuzeigen.

Im ersten Beitrag des Sonderhefts beschreiben Fleischer, Koeppen, Kenk, Klieme und Leutner aus Sicht des Koordinationsprojekts die Struktur und die zentralen Fragestellungen des Schwerpunktprogramms, welche sich vier aufeinander aufbauenden Forschungsbereichen zuordnen lassen:

1. Theoretische Kompetenzmodelle,

2. Psychometrische Modelle,

3. Messkonzepte und Messverfahren und

4. Nutzung von Informationen aus Diagnostik und Assessment.

Dabei werden der aktuelle Forschungsstand des DFG-Schwerpunktprogramms skizziert sowie offene Fragen thematisiert. Der Beitrag stellt damit gleichzeitig die thematische Einbettung für die nachfolgenden zehn Kurzbeiträge dar. 
In diesen Kurzbeiträgen werden, bezogen auf die Forschungsbereiche des Schwerpunktprogramms, Fragen und Problemstellungen diskutiert, die sich im Laufe der inhaltlichen Arbeit des Schwerpunktprogramms der letzten Jahre als besonders relevant erwiesen haben. Diese Beiträge stellen das Arbeitsergebnis eines zweitägigen Workshops im Februar 2010 der am Schwerpunktprogramm beteiligten Wissenschaftler/innen dar. Ziel des Workshops war eine vertiefende Diskussion sowie die Erarbeitung einer einheitlichen Grundposition des Schwerpunktprogramms zu den angesprochenen Fragen und Problemstellungen. Hierzu wurde zunächst in Kleingruppen diskutiert, danach wurden die Ergebnisse in Form eines Wikis zusammengetragen. Im Anschluss daran sind die einzelnen Beiträge von den unten genannten Autoren/innen erneut überarbeitet worden. Die Beiträge zwei bis elf des vorliegenden Sonderhefts können demnach nicht den Anspruch erheben, die behandelten Fragen und Problemstellungen abschließend zu beantworten beziehungsweise zu lösen. Sie stellen vielmehr in kompakter Form die Standpunkte der am Schwerpunktprogramm beteiligten Wissenschaftler/innen im Sinne von Positionspapieren dar. Gleichzeitig können die behandelten Fragestellungen nicht als voneinander unabhängig betrachtet werden. So ist beispielsweise die Wahl eines angemessenen Auflösungsgrades einer zu messenden Kompetenz zum einen eine empirische Frage. Zum anderen hängt sie jedoch auch von der angestrebten Funktion der Kompetenzmessung (formatives vs. summatives Assessment) und nicht zuletzt auch von den Adressaten der Ergebnisse der Kompetenzmessung ab.

Bezogen auf den ersten Forschungsbereich des Schwerpunktprogramms „Theoretische Kompetenzmodelle" befassen sich Wilhelm und Nickolaus in ihrem Beitrag mit der Konkretisierung des Konzeptes „Kompetenz“ und behandeln die für die Arbeiten im Schwerpunktprogramm grundlegende Frage der Abgrenzbarkeit des Kompetenzbegriffs von bewährten Konzepten wie Fähigkeit, Fertigkeit und Intelligenz. Im Anschluss gehen Leuders und Sodian der Frage nach, ob und inwiefern Kompetenzskalen, Kompetenzstufenmodelle, Kompetenzstrukturmodelle und kognitive Diagnosemodelle dazu geeignet sind, kognitive Prozesse von Lernenden zu erfassen und zu beschreiben. Neumann befasst sich mit der Frage, mit welchem Auflösungsgrad Kompetenzen modelliert werden können und unterscheidet dabei zwischen der Feingliedrigkeit (Zahl der Teilkompetenzen) und der Feinkörnigkeit (Detailliertheit der Ausprägungen) von Kompetenzmodellen. Robitzsch beschäftigt sich in seinem Beitrag mit der Frage, wie zeitlich stabil und über Situationen hinweg konstant Kompetenzen sind und welche Robustheit die hierfür verwendeten Struktur- und Niveaumodelle im Hinblick auf verschiedene Modellbestandteile (Modellparameter und Kompetenzstufenbeschreibungen) und verschiedene Analyseeinheiten (Schüler, Klassen und Populationen) aufweisen.

Mit Bezug auf den zweiten Forschungsbereich des Schwerpunktprogramms „Psychometrische Modelle“ setzen sich Hartig und Frey in ihrem Beitrag mit der Frage auseinander, inwiefern Modelle der Item-Response-Theorie (IRT) das „Mittel der Wahl“ für die Modellierung von Kompetenzen sind. Die Autoren beschreiben hierzu die Vorteile von IRT-Modellen gegenüber klassischen Ansätzen, stellen jedoch auch die Grenzen dieser Modelle dar.

Bezogen auf den dritten Forschungsbereich des Schwerpunktprogramms „Messkonzepte und Messverfahren" beschreiben Frey und Hartig in ihrem Beitrag Herausforderungen, denen mit computerbasierten Verfahren zur Messung von Kompetenzen besser begegnet werden kann als mit klassischen papier- und bleistift-basierten Verfahren. 
Hierzu zählen die Autoren komplexe Kompetenzen, Kompetenzen, die sich durch die zeitkritische Ausführung komplexer Interaktionen auszeichnen, sowie Kompetenzen, deren Ausübung an einen Computer gebunden ist.

Mit Bezug auf den vierten Forschungsbereich des Schwerpunktprogramms „Nutzung von Informationen aus Diagnostik und Assessment" befassen sich Bögeholz und Eggert in ihrem Beitrag mit der Frage, welche Rolle Kompetenzdiagnostik im Rahmen von Lehr-Lernprozessen spielt. Die Autorinnen kommen dabei zu dem Schluss, dass Kompetenzmodelle die gemeinsame Referenz für das Unterrichten sowie für formatives und summatives Assessment darstellen sollten. Im Anschluss gehen McElvany und Rjosk der Frage nach, ob und unter welchen Bedingungen Kompetenzdiagnostik negative Auswirkungen für verschiedene Akteure/innen im Bildungssystem wie Lehrkräfte, Lernende und Bildungsadministration haben kann und wie diese negativen Auswirkungen vermieden beziehungsweise verringert werden können. Pant untersucht am Beispiel nationaler und internationaler Schulleistungsstudien, die auf der Gesamtstrategie der Kultusministerkonferenz basieren, die Frage, welche Akteure/innen einen Nutzen von Kompetenzmodellen haben. Im letzten Beitrag des Sonderhefts beschäftigen sich Leiss und Rakoczy mit der Frage, wie Ergebnisse der Kompetenzdiagnostik in Forschungsprojekten sinnvoll zurückgemeldet werden können. Die Autoren schließen ihren Beitrag mit zentralen Kriterien für die Gestaltung von Rückmeldungen in Form einer „Checkliste“, anhand derer Forschungsprojekte ihre Ergebnisrückmeldungen gestalten können.

Die Beiträge des vorliegenden Sonderhefts beschreiben die Struktur und die Forschungszugänge des DFG-Schwerpunktprogramms „Kompetenzmodelle“ und spiegeln den Diskussionsstand des Schwerpunktprogramms zu grundlegenden Konzepten und Fragestellungen im Zusammenhang mit der Modellierung von Kompetenzen wider. Sie liefern damit einen breiten Überblick über die Arbeit des Schwerpunktprogramms in den letzten Jahren und zeigen gleichzeitig auch nach wie vor offene Forschungsfragen auf.

Danksagung: Diese Veröffentlichung wurde ermöglicht durch Sachbeihilfen der Deutschen Forschungsgemeinschaft (Kennz.: LE 645/11-2 und KL 1057/9-2) im Schwerpunktprogramm „Kompetenzmodelle zur Erfassung individueller Lernergebnisse und zur Bilanzierung von Bildungsprozessen“ (SPP 1293).

\section{Literatur}

Fleischer, J., Leutner, D., \& Klieme, E. (2012). Modellierung von Kompetenzen im Bereich der Bildung: Eine psychologische Perspektive (Themenheft der Psychologische Rundschau, Vol. 63(1)). Göttingen: Hogrefe Verlag.

Klieme, E., \& Leutner, D. (2006). Kompetenzmodelle zur Erfassung individueller Lernergebnisse und zur Bilanzierung von Bildungsprozessen. Beschreibung eines neu eingerichteten Schwerpunktprogramms der DFG. Zeitschrift für Pädagogik, 52, 876-903.

Klieme, E., Leutner, D., \& Kenk, M. (2010). Kompetenzmodellierung - Zwischenbilanz des DFGSchwerpunktprogramms und Perspektiven des Forschungsansatzes (56. Beiheft der Zeitschrift für Pädagogik). Weinheim. Beltz.

Koeppen, K., Hartig, J., Klieme, E., \& Leutner, D. (2008). Current issues in competence modelling and assessment. Zeitschrift für Psychologie/Journal of Psychology, 216, 61-73. 